03

\title{
Моделирование процесса резистивного динамического испарения в вакууме
}

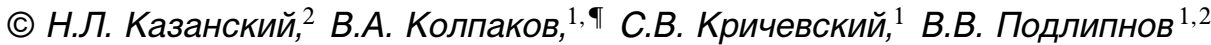 \\ ${ }^{1}$ Самарский национальный исследовательский университет им. акад. С.П. Королева, \\ 443086 Самара, Россия \\ ${ }^{2}$ ИСОИ РАН - фрилиал ФНИЦ „Кристаллография и фотоника“ РАН, \\ 443001 Самара, Россия \\ T e-mail: kolpakov683@gmail.com
}

(Поступило в Редакцию 12 апреля 2016 г. В окончательной редакции 20 марта 2017 г.)

\begin{abstract}
Представлена модель процесса резистивного динамического испарения в вакууме, учитывающая конструктивные особенности соответствующего испарителя. В рамках модели получены зависимости для определения времени нагрева материала до температуры испарения, а также динамических характеристик процесса испарения. Показано, что полученные характеристики являются негармоническими и периодически повторяющимися. Подтверждена адекватность разработанной модели физическому процессу. Установлено, что расхождение между экспериментальными и расчетными временными характеристиками движения заслонки составило не более $5 \%$. Даны рекомендации по использованию предложенной модели в технологических процессах формирования тонких пленок многокомпонентных материалов методами термовакуумного испарения.
\end{abstract}

DOI: 10.21883/JTF.2017.10.44990.1848

\section{Введение}

Методы термовакуумного испарения широко применяются в технологических процессах микро- и наноэлектроники [1,2], наноинженерии [3], дифракционной оптики и нанофотоники [4] при формировании тонких пленок одно- и многокомпонентных материалов, включая маскирующие слои, для изготовления элементов электронной и оптической компонентных баз.

Качество тонких пленок, получаемых с помощью отмеченных методов, в значительной мере зависит от типа и конструкции испарителей [5-7]. Существующие испарители, реализующие методы термовакуумного испарения, характеризуются существенными недостатками, заключающимися:

- в наличии непроизводственных потерь испаряемого вещества [8],

- в затруднении получения больших скоростей осаждения [8],

- в неоднородности потока атомов испаряемого многокомпонентного материала $[8,9]$,

- в необходимости автономного использования нескольких испарителей одновременно при напылении пленок сложного состава $[9,10]$.

От указанных недостатков свободна конструкция резистивного динамического испарителя, разгерметизация и герметизация которого осуществляются давлением пара и силами тяготения соответственно [11].

С другой стороны, требуемое качество тонких пленок обеспечивается оптимальными режимами технологических процессов формирования данных пленок. Экспериментальное определение значений параметров таких режимов представляет собой очень сложную и доро- гую операцию, поэтому актуальным является создание моделей, описывающих процессы термовакуумного испарения и учитывающих особенности конкретного типа испарителя [12-22]. Таким образом, целью настоящей работы является создание модели процесса резистивного динамического испарения в вакууме, учитывающей особенности конструкции испарителя, описанного в работе [11] и свободного от перечисленных ранее недостатков.

\section{Модель процесса резистивного динамического испарения в вакууме}

Рассмотрим процессы, протекающие при резистивном динамическом испарении в вакууме в указанном испарителе.

Поскольку процесс нагрева испаряемого материала происходит в вакууме, то теплопередача осуществляется посредством излучения спирали нагревателя 3 за счет протекания через него электрического тока (рис. 1). Тепло, переданное корпусу испарителя, аналогичным образом частично идет на испарение, а частично излучается в окружающее пространство вакуумной камеры. В процессе нагревания при достижении температуры испарения происходит накопление паров испаряемого материала, сопровождающееся повышением давления в замкнутой области. Определение времени нагрева испаряемого материала до температуры испарения $\left(\tau_{\text {set }}\right)$ осуществляется с помощью решения системы уравнений теплового баланса [23], которые для рассматриваемого 


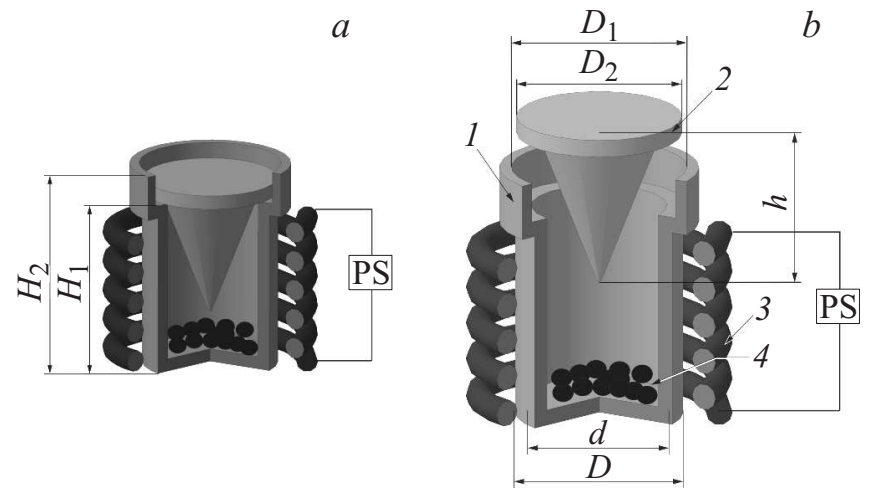

Рис. 1. Схема конструкции испарителя в состоянии, когда заслонка закрыта $(a)$, и в состоянии, когда заслонка открыта (b) [11]: 1 - полый корпус, 2 - заслонка, 3 - проволочный нагреватель, 4 - испаряемый материал.

случая имеют вид

$$
\begin{gathered}
W d \tau=C_{\mathrm{sp}} m_{\mathrm{sp}} d T_{\mathrm{sp}}+\varepsilon_{\mathrm{sp}} \sigma_{0} T_{\mathrm{sp}}^{4} \pi d_{\mathrm{sp}} l_{\mathrm{sp}} d \tau, \\
K_{\mathrm{int}} \varepsilon_{\mathrm{gr}} Q_{\mathrm{sp}} d \tau=C_{\mathrm{gr}} m_{\mathrm{gr}} d T_{\mathrm{gr}}+\varepsilon_{\mathrm{gr}} \sigma_{0} T_{\mathrm{gr}}^{4} S_{\mathrm{gr}} d \tau,
\end{gathered}
$$

где $Q_{\mathrm{sp}}-$ мощность излучения нагретой спирали, равная $Q_{\mathrm{sp}}=\varepsilon_{\mathrm{sp}} \sigma_{0} \pi d_{\mathrm{sp}} l_{\mathrm{sp}} ; W-$ мощность, подводимая к нагревательной спирали, $\mathrm{W} ; d \tau$ - приращение времени, s; $C_{\mathrm{sp}}$ - теплоемкость материала спирали нагревателя, $\mathrm{J} /(\mathrm{kg} \cdot \mathrm{K}) ; m_{\mathrm{sp}}$ - масса материала спирали, $\mathrm{kg} ; \varepsilon_{\mathrm{sp}}-$ коэффициент черноты вольфрамовой спирали; $\sigma_{0}-$ постоянная Стефана-Больцмана, $\mathrm{W} /\left(\mathrm{m} \cdot \mathrm{K}^{4}\right) ; d_{\mathrm{sp}}$ - диаметр спирали, $\mathrm{m} ; l_{\mathrm{sp}}$ - длина спирали нагревателя, $\mathrm{m}$; $T_{\mathrm{sp}}$ - температура спирали, $\mathrm{K}, C_{\mathrm{gr}}$ - теплоемкость испарителя, равная $C_{\mathrm{gr}}=720 \mathrm{~J} /(\mathrm{kg} \cdot \mathrm{K}) \quad[24] ; \quad m_{\mathrm{gr}}-$ масса материала испарителя, $\mathrm{kg} ; T_{\mathrm{gr}}-$ температура испарителя, K; $\varepsilon_{\mathrm{gr}}-$ степень черноты графитового корпуса испарителя, равная 0.8 [24-27]; $S_{\mathrm{gr}}-$ площадь поверхности испарителя, равная $S_{\mathrm{gr}}=0.00335 \mathrm{~m}^{2}$; $K_{\text {int }}$ - интегральный коэффициент, учитывающий полезную часть излучаемой энергии, поглощаемой испарителем и зависящий от толщины проволоки, параметров испарителя, параметров намотки на него нагревательной спирали, плотности витков, установки дополнительных теплоотражающих экранов или их отсутствия. Коэффициент $K_{\text {int }}$ определяется методом геометрического построения или $3 d$-моделированием для поверхностей сложных форм, описанным в работах [27-29].

Решение системы уравнений (1) и (2) относительно величины $\tau$ осуществлено с помощью метода разложения на простейшие дроби [30], а выполнение вычислений реализовано в программном пакете Maple. Результатом решения является аналитическое выражение вида

$$
\begin{aligned}
\tau_{\mathrm{set}}= & \frac{0.25 C_{\mathrm{gr}} m_{\mathrm{gr}}\left(\frac{Q_{\mathrm{sp}}}{\sigma_{0} S_{\mathrm{gr}}}\right)^{1 / 4}}{\varepsilon_{\mathrm{gr}} Q_{\mathrm{sp}}} \\
& \times\left(\ln \left(\frac{T_{\mathrm{isp}}+\left(\frac{Q_{\mathrm{sp}}}{\sigma_{0} S_{\mathrm{gr}}}\right)^{1 / 4}}{T_{\mathrm{isp}}-\left(\frac{Q_{\mathrm{sp}}}{\sigma_{0} S_{\mathrm{gr}}}\right)^{1 / 4}} \frac{T_{0}-\left(\frac{Q_{\mathrm{sp}}}{\sigma_{0} S_{\mathrm{gr}}}\right)^{1 / 4}}{T_{0}+\left(\frac{Q_{\mathrm{sp}}}{\sigma_{0} S_{\mathrm{gr}}}\right)^{1 / 4}}\right)\right. \\
& \left.+2\left(\arctan \frac{T_{\mathrm{isp}}}{\left(\frac{Q_{\mathrm{sp}}}{\sigma_{0} S_{\mathrm{gr}}}\right)^{1 / 4}}-\arctan \frac{T_{0}}{\left(\frac{Q_{\mathrm{sp}}}{\sigma_{0} S_{\mathrm{gr}}}\right)^{1 / 4}}\right)\right)
\end{aligned}
$$

Высокая теплопроводность графита и достаточно тонкие стенки полости испарителя $(1-1.5 \mathrm{~mm})$ позволяют считать процесс нагрева испаряемого материала безынерционным и принять его температуру равной температуре испарителя. При проведении вычислительных и натурных экспериментов использовался теллурид кадмия $(\mathrm{CdTe})$ температура испарения которого при давлении 1.33 Ра равна $900 \mathrm{~K}$ [31-33]. Тогда для достижения такой температуры, согласно выражениям (1)-(3) необходима мощность источника питания, равная $400 \mathrm{~W}$. Подставляя в (3) соответствующее численное значение параметра $m_{\mathrm{gr}}=0.012 \mathrm{~kg}$, получим $\tau_{\mathrm{set}}=88 \mathrm{~s}$. По прошествии данного времени вещество в испарителе нагревается до температуры испарения (давление превышает $1.33 \mathrm{~Pa}$ ). В дальнейшем происходит накопление паров испаряемого материала в корпусе испарителя посредством сублимации. Рассмотрим данный исследуемый процесс.

Итак, в единицу времени происходит испарение $d m$ материала. Тогда, использовав уравнение теплового баланса (2) при неизменной температуре и определив количество теплоты, расходуемое на процесс испарения $Q_{\text {isp }}=K_{\mathrm{int}} \varepsilon_{\mathrm{gr}} Q_{\mathrm{sp}}-\varepsilon_{\mathrm{gr}} \sigma_{0} T_{\mathrm{gr}}^{4} S_{\mathrm{gr}}$, можно записать следующее соотношение [34]:

$$
\frac{d m}{d \tau}=\frac{Q_{\text {isp }}}{L},
$$

где $L$ - удельная теплота сублимации, $\mathrm{J} / \mathrm{kg}$ [35].

Используя зависимость (4), определим количество атомов испаряемого вещества, перешедшего в газообразное состояние за единицу времени:

$$
\frac{d N_{\mathrm{isp}}}{d \tau}=\frac{Q_{\mathrm{isp}} N_{a}}{L M},
$$

где $N_{a}$ - число Авагадро, mol ${ }^{-1} ; M-$ приведенная молярная масса $\mathrm{CdTe}, \mathrm{g} / \mathrm{mol}$.

Изменение давления газа за единицу времени внутри испарителя зависит от температуры и изменения концентрации атомов в объеме полости испарителя. Отмеченное изменение концентрации обусловлено одновременным протеканием процессов накопления в объеме полости испарителя атомов материала в газообразном 
состоянии и истечения данных атомов из указанного объема через зазор, образующийся в случае, когда сила давления паров испаряемого материала начинает превышать силу прижима заслонки к торцевой поверхности корпуса испарителя. Тогда, согласно уравнению состояния идеального газа [36-37], можно записать

$$
\frac{d P}{d \tau}=\frac{d N_{\text {in }}}{d \tau} \frac{k T}{V}
$$

где

$$
\frac{d N_{\text {in }}}{d \tau}=\frac{d N_{\text {isp }}}{d \tau}-\frac{d N_{\text {out }}}{d \tau}
$$

- изменение концентрации атомов газа внутри испарителя за единицу времени, $d N_{\text {out }} / d \tau-$ количество атомов испаряемого материала в газообразном состоянии, покинувших объем испарителя за единицу времени, $k$ - постоянная Больцмана, $\mathrm{J} / \mathrm{K} ; V$ - объем полости испарителя, $\mathrm{m}^{3}$.

Характер истечения газа через указанный ранее зазор зависит от давления газа и определяется критерием Кнудсена $\left(K_{n}\right)$, равным отношению среднего свободного пробега атомов газа $\left(\lambda=k T /\left(\sqrt{2} P \pi D_{0}^{2}\right)\right)$ к характерному размеру сосуда $\left(d_{V}\right)$ [38-41]. Рассматриваемый случай соответствует молекулярному истечению газа, так как выполняется неравенство $\lambda \gg d_{V}[41,42]$. Используя формулу Ленгмюра [43], найдем скорость истечения атомов испаряемого материала через отверстие (кольцевой зазор) площадью $S$ по формуле

$$
\left(\frac{\Delta m}{\Delta \tau}\right)_{M}=\frac{P S K}{\sqrt{\frac{2 \pi R T}{M}}}
$$

где $K$ - коэффициент Клаузинга [44], $R$ - универсальная газовая постоянная, $\mathrm{J} / /(\mathrm{mol} \cdot \mathrm{K})$. Следовательно, скорость истечения пара за единицу времени через кольцевой зазор с изменяющимся сечением, зависящим от высоты поднятия заслонки $(x)$, записывается в виде

$$
\frac{d N_{\mathrm{out}}}{d \tau}=\frac{N_{a} P S(x) K(x)}{\sqrt{2 \pi R T M}} .
$$

Таким образом, подставляя в выражение (6) формулы (5), (7), можно выразить зависимость изменения давления паров внутри испарителя от времени и высоты подъема заслонки (рис. 1, 2):

$$
\frac{d P}{d \tau}=\left(\frac{Q_{\text {isp }} N_{a}}{L M}-\frac{N_{a} P(\tau, x) S(x) K(x)}{\sqrt{2 \pi R T M}}\right) \frac{k T}{V(x)},
$$

где

$$
\begin{gathered}
S(x)=2 \pi d / 2 x \sin \alpha-\pi \sin \alpha^{2} x^{2} \cos \alpha \\
V(x)=\pi\left(\frac{d}{2}\right)^{2}\left(H_{1}-\frac{1}{3} \frac{(d \cos \alpha}{2 \sin \alpha}\right)+\pi\left(\frac{d}{2}\right)^{2} x
\end{gathered}
$$

и

$K(x)=\left\{\begin{array}{l}\frac{l}{x \sin \alpha}>0.75: K=\frac{1+0.8 \frac{l}{x \sin \alpha}}{1+\frac{1.9 l}{x \sin \alpha}+0.6\left(\frac{l}{x \sin \alpha}\right)^{2}} \\ \frac{l}{x \sin \alpha}<0.75: K=\frac{1}{1+l / x \sin \alpha}\end{array}\right\}$



Рис. 2. Схема геометрического расчета площади проходного сечения.

вычисляются в соответствии со схемой геометрического расчета (см. рис. 2), а также по эмпирическим формулам Кеннарда [45] (величина $K(x)$, где $l$ - длина канала, ограничивающего выход молекул; $\alpha$ - половина угла при вершине осевого сечения конуса заслонки).

Для решения дифференциального уравнения (8) его необходимо дополнить уравнением движения второго порядка, которое можно составить, используя баланс сил, действующих на заслонку испарителя, а также зависимость подъемной силы от координаты и давления:

$$
m \ddot{x}=P(\tau, x) \pi(d / 2)^{2}-m_{Z} a_{G},
$$

где $a_{G}$ - ускорение свободного падения, $\mathrm{m} / \mathrm{s}^{2} ; m_{Z}-$ масса заслонки испарителя, g. Решая систему дифференциальных уравнений (8) и (9), можно легко определить основные временные характеристики процесса резистивного динамического испарения: зависимости давлениявнутри испарителя, высоты подъема заслонки, коэффициента проводимости Клаузинга, площади проходного сечения и числа атомов, истекающих из испарителя от времени.

Решение системы дифференциальных уравнений (8), (9) осуществлено численно с помощью метода конечных разностей [46] и реализовано в математическом пакете Maple при следующих граничных и начальных условиях:

$$
\left\{\begin{array}{l}
a(0, \tau)=0, \\
v(0, \tau)=0, \\
x(0,0)=0, \quad x \geq 0 \text { при } \tau>0, \\
\left.S(0, \tau)=0, \quad 0 \leq S \leq \pi(d / 2)^{2} \text { при } \tau>0\right) .
\end{array}\right.
$$

В процессе решения учитывались следующие обстоятельства. В случае, когда величина $x$ становилась 
меньше нуля, переменным $v$ и $a$, получаемым в ходе решения уравнения (9), присваивались нулевые значения. Физически это означает полное опускание заслонки на торцевую поверхность корпуса испарителя и нахождение ее в состоянии покоя до тех пор, пока давление внутри замкнутой полости испарителя снова не достигнет порогового значения, при котором заслонка придет в движение. Ограничение вычисляемой площади проходного сечения $S$ величиной $\pi(d / 2)^{2}$ установлено исходя из того, что в процессе подъема заслонки площадь $S$ увеличивается (см. зависимость (8) и относящуюся к ней формулу для определения величины $S$ ) и, начиная с некоторой высоты подъема $x$, становится больше площади отверстия испарителя. Следовательно, поток испаряемых атомов ограничивает сечение самого испарителя, площадь которого равна $S=\pi(d / 2)^{2}$.

\section{Результаты и обсуждение}

Для оценки адекватности разработанной модели физическому процессу проводились вычислительный и натурный эксперименты, в которых в качестве испаряемого материала использовался теллурид кадмия (CdTe) с молярной массой $M=240 \mathrm{~g} / \mathrm{mol}$ и теплотой сублимации, равной $L=65210 \mathrm{~J} / \mathrm{mol}[35,36]$. Согласно результатам расчета, к спирали нагревателя испарителя подводилась электрическая мощность, равная $400 \mathrm{~W}$. Процесс испарения осуществлялся с помощью исследуемого испарителя, установленного в рабочей камере вакуумной установки УВН-2М-1. В полый корпус 1 испарителя (рис. 1) загружались кристаллы химически чистого CdTe массой, равной $m=2 \mathrm{~g}$. Рабочая камера вакуумной установки откачивалась до остаточного давления, равного $P_{0}=2 \cdot 10^{-4} \mathrm{~Pa}$. Температура корпуса испарителя определялась с помощью инфракрасного пирометра СЕМ DT-8865. Процесс испарения регистрировался высокоскоростной видеокамерой VS-FAST/CG6 компании НПК „Видеоскан“ (Россия) со скоростью до 1000 fps. Определение временных характеристик движения заслонки осуществлялось посредством покадровой обработки видеозаписи, полученной с помощью указанной видеокамеры.

На рис. 3 представлены расчетные временные характеристики процесса резистивного динамического испарения CdTe в вакууме, анализ которых позволяет выделить следующие особенности рассматриваемого процесса. В интервале времени $0<\tau<0.04$ s давление паров меньше давления, уравновешивающего вес заслонки, равного $P_{Z}=113 \mathrm{~Pa}$ (рис. 3, $a$ ), и рост величины $P$ происходит линейно, поскольку заслонка находится в состоянии покоя, а, следовательно, отсутствует выход паров из испарителя. С момента времени $\tau=0.04 \mathrm{~s}$ давление внутри испарителя превышает величину давления, оказываемого заслонкой на газ, в результате чего возникает сила, приводящая заслонку в движение, характеризуемое положительным перемещением (рис. 3, b). После открытия заслонки рост давления продолжается, но уже нелинейно. Такое поведение зависимости $P=f(\tau)$ объясняется тем, что в начальный момент подъема заслонки количество испаряющихся атомов превышает количество атомов, истекающих из испарителя (рис. $3, e$ ) в виду нелинейного закона изменения проходного сечения (рис. $3, d)$ и коэффициента Клаузинга (рис. 3,c). Согласно проведенным расчетам, пропорционально росту давления продолжается и рост ускорения движения заслонки вверх, величина которого достигает своего максимального значения $a=7.6 \mathrm{~m} / \mathrm{s}^{2}$ при $\tau=0.08 \mathrm{~s}$, а также $P=201 \mathrm{~Pa}$ (рис. 3, $a$ ). После указанного момента времени коэффициент Клаузинга принимает значения $K>0.4$ (рис. $3, c$ ), при которых количество атомов, истекающих из испарителя, превышает число испаряющихся атомов, что приводит к уменьшению давления паров, а следовательно, и к уменьшению ускорения движения заслонки вверх в области $0.08<\tau<0.12$ s. Далее, величина $a$ принимает отрицательные значения в диапазоне $0.12<\tau<0.175 \mathrm{~s}$, что приводит к замедлению движения заслонки вверх, а затем при $0.175 \leq \tau \leq 0.255$ s к ее опусканию (рис. $3, b$ ). Сравнительный анализ зависимостей $P=f(\tau), S=f(\tau)$ и $N=f(\tau)$ показывает, что в диапазоне $0.12 \leq \tau \leq 0.175 \mathrm{~s}$ заслонка практически не оказывает сопротивления истечению паров из испарителя, о чем свидетельствуют минимальные изменения давления, ускорения и величины $N$. Построение зависимости $a=f(\tau)$ на указанном ранее промежутке времени свидетельствует о равноускоренном падении заслонки. Однако ее ускорение при этом меньше ускорения свободного падения $\left(a_{G}\right)$ и равно $6.5 \mathrm{~m} / \mathrm{s}^{2}$, что объясняется противодействием потока испаряющихся атомов. При $\tau \geq 0.23 \mathrm{~s}$ движение заслонки вниз начинает замедляться, что сопровождается резким уменьшением коэффициента Клаузинга (рис. 3,c) и площади проходного сечения (рис. $3, d$ ). Движение заслонки вниз продолжается вплоть до полного перекрытия проходного сечения и опускания ее на торцевую поверхность корпуса испарителя в момент времени $\tau=0.255 \mathrm{~s}$ истечение паров прекращается (рис. 3,e). В данный момент времени заслонка становится неподвижной. Через некоторое время давление внутри испарителя вновь становится выше давления паров, уравновешивающего вес заслонки и процесс ее движения, а также истечения паров из испарителя циклически повторяется.

Интегрируя зависимость $N=f(\tau)$ за время одного цикла, получим количество атомов испаренного материала, истекающих из испарителя за одно открытие заслонки, которое, согласно расчету, равно $N=0.17 \cdot 10^{16}$ и соответствует массе $m=6.9 \cdot 10^{-7} \mathrm{~g}$. Таким образом, при загрузке $2 \mathrm{~g}$ испаряемого вещества произойдет 2898 открытий заслонки за время $725 \mathrm{~s}$, после чего вещество полностью испарится, а ее колебания прекратятся.

Исследования методами растровой электронной и сканирующей зондовой микроскопии тонких пленок CdTe, сформированных с помощью рассматриваемого испарителя и представленных результатов моделирования, подтвердили преимущества таких пленок перед пленками, получаемыми традиционными методами резистивного испарения в вакууме [47]. 



Рис. 3. Временны́е характеристики процесса резистивного динамического испарения СdТе в вакууме: $a-$ зависимость давления паров испаряемого материала внутри испарителя от времени (сплошная линия - давление паров, уравновешивающее вес заслонки), $b$ - зависимость высоты подъема заслонки от времени, $c-$ зависимость коэффициента Клаузинга от времени, $d-$ зависимость площади проходного сечения от времени, $e$ - зависимость интенсивности выхода атомов испаряемого материала, истекающих из испарителя, от времени. - - расчетные значения, + - экспериментальные значения, полученные в результате обработки видеозаписи высокоскоростной видеокамеры VS-FAST/CG6.

Сравнение результатов проведенных вычислительного и натурного экспериментов показывает высокое соответствие временных характеристик движения заслонки, полученных посредством обработки видеозаписи, расчетным данным (рис. $3, b$ ). Расхождение между экспериментальными и расчетными данными составило не более $5 \%$, что подтверждает высокую точность разработанной модели.

\section{Заключение}

В настоящей работе предложена модель процесса резистивного динамического испарения в вакууме, адекватная физическому процессу, учитывающая особенности конструкции испарителя, описанного в работе [11] и свободного от недостатков, характерных для существующих аналогов. В рамках модели получены зависимости для определения времени нагрева материала до температуры испарения, а также динамических характеристик процесса испарения. Полученные характеристики позволяют в заданный момент времени оценивать давление паров испаряемого материала внутри испарителя, ускорение, скорость движения заслонки, высоту ее подъема, коэффициент Клаузинга, площадь проходного сечения, а также количество атомов испаряемого материала, истекающих из испарителя, что было затруднительным до появления настоящей работы. С помощью проведенного вычислительного эксперимента установлено, что характер движения заслонки и выхода паров из корпуса испарителя является негармоническим, периодически повторяющимся, при этом истечение атомов испаренного материала происходит импульсно в количестве $N=0.17 \cdot 10^{16}$ за одно открытие заслонки. Показано, что при электрической мощности, подводимой к спирали нагревателя испарителя, равной $400 \mathrm{~W}$, диапазоны изменения давления внутри испарителя, ускорения движения заслонки, высоты ее подъема, коэффи- 
циента Клаузинга, а также площади проходного сечения составили: $2 \cdot 10^{-4} \leq P \leq 201 \mathrm{~Pa}, 0 \leq a \leq 7.6 \mathrm{~m} / \mathrm{s}^{2}$, $0 \leq x \leq 0.024 \mathrm{~m}, 0 \leq K \leq 0.85$ и $0 \leq S \leq 0.00033 \mathrm{~m}^{2}$ соответственно. Проведение натурного эксперимента показало, что расхождение между экспериментальными и расчетными временными характеристиками движения заслонки составило не более 5\%. Указанное подтверждает высокую точность разработанной модели. Применение предложенной модели позволит повысить качество тонких пленок многокомпонентных материалов, формируемых методами термовакуумного испарения, посредством улучшения их стехиометрического состава. В связи с этим логическим продолжением проделанной работы будет исследование свойств тонких пленок многокомпонентных материалов, полученных с использованием исследуемого испарителя на основе представленных результатов моделирования.

Работа выполнена при поддержке грантов президента Российской Федерации для поддержки молодых российских ученых - докторов наук МД-5205.2016.9 и Российского фонда фундаментальных исследований (проект № 16-07-00494 A).

\section{Список литературы}

[1] Jaeger R.C. Film Deposition: Introduction to Microelectronic Fabrication (2nd ed.). N. J.: Prentice Hall, 2002. 332 p.

[2] Aldridge J.S., Cleland A.N., Knobel R., Schmidt D.R., Yung C.S. In International Symposium on Microelectronics and MEMS. International Society for Optics and Photonics. 2001. P. 11-21.

[3] Dai Z.R., Pan Z.W., Wang Z.L. // Advanced Functional Materials. 2003. Vol. 13. N 1. P. 9-24.

[4] Diffractive nanophotonics / Ed. by V.A. Soifer. London: Taylor and Francis, 2014. 704 p.

[5] Amirhaghi S., Craciun V., Craciun D., Elders J., Boyd I.W. // Microelectronic engineering. 1994. Vol. 25. N 2-4. P. 321326.

[6] Botman A. et al. // Nanotechnology. 2006. Vol. 17. N 15. P. 3779.

[7] Грицюк Б.Н. и др. // ЖТФ. 2001. Т. 71. Вып. 9. С. 54-57.

[8] Mattox D.M. // Products Finishing-Cincinnati. 1999. N 63. P. $48-58$.

[9] Suryanarayana C., Yoo S.H., Groza J.R. // J. Mater. Sci. Lett. 2001. Vol. 20. N 24. P. 2179-2181.

[10] Wu Y.L. et al. // Thin Solid Films. 1989. Vol. 168. N 1. P. 113122.

[11] Сойфер В.А., Казанский Н.Л., Колпаков В.А., Колпаков А.И., Подлипнов В.В. // Патент RU2348738. 2009.

[12] Powell A. et al. // Metallurgical and Materials Transactions A. 2001. Vol. 32. N 8. P. 1959-1966.

[13] Frezzotti A. // European J. Mechanics B/Fluids. 2007. Vol. 26. N 1. P. 93-104.

[14] Yan H. et al. // Materials Research Bulletin. 2009. Vol. 44. N 10. P. 1954-1958.

[15] Shah N.M. et al. // Thin Solid Films. 2009. Vol. 517. N 13. P. 3639-3644.

[16] Cochelin E. et al. // J. Thermal Spray Technology. 1999. Vol. 8. N 1. P. 117-124.
[17] Tachibana K., Nishida M., Harima H., Urano Y. // J. Phys. D: Appl. Phys. 1984. Vol. 17. N 8. P. 1727.

[18] Elimelech M., Gregory J., Jia X. Particle deposition and aggregation: measurement, modelling and simulation. Butterworth-Heinemann, 1995. 440 p.

[19] Moarrefzadeh A., Branch M. // Wseas Transactions on Applied and Theoretical Mechanics. 2012. Vol. 7. N 2. P. 106 111.

[20] Lalitha S., Sathyamoorthy $R$., Senthilarasu S., Subbarayan A., Natarajan K. // Solar Energy Materials and Solar Cells. 2004. Vol. 82. N 1. P. 187-199.

[21] Tobias J.S., Birkmire R.W., Doyle F.J. // AIChE J. 2005. Vol. 51. N 3. P. 878-894.

[22] Adachi Sadao, Toshifumi Kimura // Japan. J. Appl. Phys. 1993. Vol. 32. N 8. P. 3496.

[23] Labuntsov D.A., Kryukov A.P. // Inter. J. Heat and Mass Transfer. 1979. Vol. 22. N 7. P. 989-1002.

[24] Isaacs L.L., Wang W.Y. // Therm. Conduct. 1981. N 17. P. 55 61.

[25] Маркелов Н.В., Волга В.И., Бучнев Л.М. // Журн. физ. химии. 1973. Т. 47. Вып. 7. С. 1824-1827.

[26] Блох А.Г. Основы теплообмена излучением. М.: Госэнергоиздат, 1962. $332 \mathrm{c.}$

[27] Howell J.R., Menguc M.P., Siegel R. Thermal radiation heat transfer. London: Taylor and Francis, 2010. 857 p.

[28] Powell K.M., Edgar T.F. // Chem. Engin. Sci. 2012. Vol. 71. P. $138-145$.

[29] Wu Z., Li S., Yuan G., Lei D., Wang Z. // Appl. Energy. 2014. Vol. 113. P. 902-911.

[30] Arfken G.B., Weber H.J., Harris F.E. Mathematical methods for physicists: a comprehensive guide (7th ed.). N.Y: Academic Press, 2012. 717 p.

[31] Toma O., Ion L., Girtan M., Antohe S. // Solar Energy. 2014. Vol. 108. P. 51-60.

[32] Brus V.V., Solovan M.N., Maistruk E.V., Kozyarskii I.P., Maryanchuk P.D., Ulyanytsky K.S., Rappich J. // Phys. Sol. Stat. 2014. Vol. 56. N 10. P. 1947-1951.

[33] Боброва Е.А., Клевков Ю.В., Черноок С.Г., Сентюрина Н.Н. // ФТП. 2014. Т. 48. Вып. 3. С. 421-427.

[34] Crout D. // J. Mathematics and Physics. 1936. Vol. 15. N 1. P. $1-54$.

[35] Kauzmann $W$. Kinetic theory of gases. N.Y: Dover Publications, 2012. 249 p.

[36] Сивухин Д.В. Общий курс физики. Т. 2. Термодинамика и молекулярная физика (5-е изд.). М.: Физматлит, 2005. $544 \mathrm{c}$.

[37] Wu Y.S. // Phys. Rev. Lett. 1994. Vol. 73. N 7. P. 922-925.

[38] Farrow R.F.C. // J. Phys. D: Appl. Phys. 1974. Vol. 7. N 17. P. 2436

[39] Oh C.K., Oran E.S., Sinkovits R.S. // J. Thermophysics and Heat Transfer. 1997. Vol. 11. N 4. P. 497-505.

[40] Hotyst R., Litniewski M. // The J. Chem. Phys. 2009. Vol. 130. N 7. P. 074707.

[41] Stops D.W. // J. Phys. D: Appl. Phys.

[42] Печатников Ю.М. // ЖТФ. 2003. Т. 73. Вып. 8. С. 40-44.

[43] Любитов Ю.Н. // УФН. 1976. Т. 119. Вып. 8. С. 641-688.

[44] Winterbottom W.L., Hirth J.P. // The J. Chem. Phys. 1962. Vol. 37. N 4. P. 784-793.

[45] Kennard E.H. Kinetic Theory of Gases. N. Y.: McGraw-Hill, 1938. 496 p.

[46] Smith G.D. Numerical solution of partial differential equations: finite difference methods. Oxford: Oxford university press, $1985.339 \mathrm{p}$.

[47] Колпаков В.А., Кричевский С.В., Подлипнов В.В. // ПТЭ. 2017. № 2. C. 1-4. 